\title{
Protective Effect of Isoliquiritigenin against Ethanol-Induced Hepatic Steatosis by Regulating the SIRT1-AMPK Pathway
}

\author{
Ann-Yae $\mathrm{Na}^{1}$, Eun-Ju Yang', Ju Mi Jeon', Sung Hwan $\mathrm{Ki}^{2}{ }^{1}$, Kyung-Sik Song' and Sangkyu Lee' \\ 'BK21 Plus KNU Multi-Omics Based Creative Drug Research Team, College of Pharmacy, Research Institute of \\ Pharmaceutical Sciences, Kyungpook National University, Daegu, Korea \\ ${ }^{2}$ College of Pharmacy, Chosun University, Gwangju, Korea
}

\begin{abstract}
Ethanol-induced fat accumulation, the earliest and most common response of the liver to ethanol exposure, may be involved in the pathogenesis of liver diseases. Isoliquiritigenin (ISL), an important constituent of Glycyrrhizae Radix, is a chalcone derivative that exhibits antioxidant, anti-inflammatory, and phytoestrogenic activities. However, the effect of ISL treatment on lipid accumulation in hepatocytes and alcoholic hepatitis remains unclear. Therefore, we evaluated the effect and underlying mechanism of ISL on ethanol-induced hepatic steatosis by treating AML-12 cells with $200 \mathrm{mM}$ ethanol and/or ISL $(0 \sim 50 \mu \mathrm{M})$ for $72 \mathrm{hr}$. Lipid accumulation was assayed by oil red O staining, and the expression of sirtuin1 (SIRT1), sterol regulatory element-binding protein-1c (SREBP-1c), AMP-activated protein kinase (AMPK), and peroxisome proliferator-activated receptor alpha (PPAR $\alpha$ ) was studied by western blotting. Our results indicated that ISL treatment upregulated SIRT1 expression and downregulated SREBP-1c expression in ethanol-treated cells. Similarly, oil red O staining revealed a decrease in ethanolinduced fat accumulation upon co-treatment of ethanol-treated cells with 10,20 , and $50 \mu \mathrm{M}$ of ISL. These findings suggest that ISL can reduce ethanol induced-hepatic lipogenesis by activating the SIRT1-AMPK pathway and thus improve lipid metabolism in alcoholic fatty livers.
\end{abstract}

Key words: Isoliquiritigenin, AML-12 cells, Ethanol, Hepatic steatosis, NAFLD

\section{INTRODUCTION}

Alcoholic fatty liver disease (AFLD) is a leading cause of liver diseases worldwide. Hepatic steatosis, the benign form of AFLD, is the most general response to chronic heavy alcohol consumption (1). The development of alcoholic steatosis involves alcohol and acetaldehyde-induced direct hepatocyte injury and death caused by increased reactive oxygen and nitrogen species production, lipid peroxidation, and oxidative DNA injury (2). Moreover, by promoting hepatic lipid accumulation, alcohol plays a

Correspondence to: Sangkyu Lee, College of Pharmacy, Kyungpook National University, Daegu 41566, Korea

E-mail: sangkyu@knu.ac.kr

This is an Open-Access article distributed under the terms of the Creative Commons Attribution Non-Commercial License (http:// creativecommons.org/licenses/by-nc/3.0) which permits unrestricted non-commercial use, distribution, and reproduction in any medium, provided the original work is properly cited. major role in activating hepatic inflammatory processes and reprograming lipid metabolism (3-5).

Several pathways are involved in the pathogenesis of ethanol-induced liver disease. One of the central pathways involves the induction of cytochrome P450 2E1 by ethanol, leading to the induction of lipid peroxidation in hepatocytes (6). A second pathway involves the regulation of transcription factors associated with fat metabolism by ethanol. Ethanol also affects the activities of enzymes involved in energy metabolism including AMPactivated protein kinase (AMPK) and sirtuin-1 (SIRT1). SIRT1 plays a vital role in the regulation of systemic energy production and steroid homeostasis in the liver (7). Previous studies have indicated that acetaldehyde and acetate, two major ethanol metabolites, significantly inhibit SIRT1 expression and activity in cultured macrophage cell lines (8). In addition, it was suggested that shRNA-mediated knockdown of SIRT1 has a critical role in the development of alcoholic steatosis (8). Ethanol-mediated 
<smiles>O=C(/C=C/c1ccc(O)cc1)c1ccc(O)cc1O</smiles>

Fig. 1. Chemical structure of isoliquiritigenin.

dysregulation of hepatic AMPK, a lipid regulator, is one of the major mechanisms in the pathogenesis of AFLD (911), as the impaired AMPK signaling accelerates lipid accumulation and inhibits lipid catabolism ultimately leading to AFLD development in animals (12).

The proven effectiveness of herbal therapies and traditional Chinese medicines against various diseases has led to an increase in their usage in western countries (13). Isoliquiritigenin (ISL, Fig. 1) is a natural flavonoid with a chalcone structure $\left(4,2^{\prime}, 4^{\prime}\right.$-trihydroxychalcone) isolated from licorice (Glycyrrhiza uralensis) roots (14). It has various biological properties, including anti-inflammatory, antioxidant, anti-platelet aggregation, vasorelaxant, and estrogenic properties (2,15-18). ISL is also a potent mitotic inhibitor and an inducer of apoptosis and might therefore have a significant antitumor activity $(19,20)$. Furthermore, studies have shown that ISL can induce autophagy in many cancer cell lines (21). However, the effect of ISL on hepatic SIRT1-AMPK signaling in ethanol-induced fatty liver disease remains largely unknown.

Therefore, it is of interest to determine whether ISL has beneficial effects on SIRT1 and AMPK activity. We hypothesized that ISL may improve the ethanol-induced, SIRT1-AMPK signaling-mediated dysregulation of lipid metabolism. In this study, we showed that ISL potently increased both SIRT1 and AMPK phosphorylation, which, in turn, reduced the triglyceride (TG) accumulation in alcoholic steatosis. Thus, this study provides a novel mechanistic insight into AFLD pathogenesis and suggests that ISL may be a suitable candidate for the pharmacological intervention of alcoholic steatosis.

\section{MATERIALS AND METHODS}

Materials. ISL ( $>95 \%$ pure), a yellow, water-insoluble powder, was isolated from the ethanol extract of Glycyrrhizae Radix in our previous study (22). A stock solution (100 mM) was prepared in dimethyl sulfoxide (DMSO, Sigma-Aldrich, St. Louis, USA), aliquoted, and stored at $-20^{\circ} \mathrm{C}$ until use. For all experiments, the final concentrations of the test compound were prepared by diluting the stock with medium. Control cells were treated with the carrier solvent $(<0.1 \%$ DMSO).
Cell culture and treatment. AML-12 cell line was purchased from the American Type Culture Collection (ATCC, Manassas, VA, USA), plated at a density of $3 \times$ $10^{5}$ cells per well in $60 \mathrm{~mm}$ dishes, and grown to $70 \sim 80 \%$ confluency. The cells were maintained in Dulbecco's modified Eagle's medium/nutrient mixture F-12 containing $10 \%$ fetal bovine serum (Hyclone, Logan, UT, USA), 50 units $/ \mathrm{mL}$ penicillin and $50 \mathrm{mg} / \mathrm{mL}$ streptomycin (Gibco, Waltham, MA, USA), $0.005 \mathrm{mg} / \mathrm{mL}$ insulin (Gibco) and $40 \mathrm{ng} / \mathrm{mL}$ dexamethasone (Sigma-Aldrich) at $37^{\circ} \mathrm{C}$ with $5 \% \mathrm{CO}_{2}$. ISL was dissolved in DMSO, diluted with phosphate-buffered saline (PBS, Gibco), and added to the cells. The cells were then incubated at $37^{\circ} \mathrm{C}$ for the indicated time period and washed twice with ice-cold PBS before sample preparation.

Cell viability assay. The effect of ethanol and ISL treatment on cell viability was examined in a dose-dependent manner by the water-soluble tetrazolium (WST)-1 assay (Roche, Mannheim, Germany), according to the manufacturer's instructions. Briefly, cells were seeded in 96-well plates $\left(3 \times 10^{5}\right.$ cells per well), cultured for $24 \mathrm{hr}$, and treated with various concentrations of ethanol $(0 \sim 500$ $\mathrm{mM})$ or ISL $(0 \sim 200 \mu \mathrm{M})$ in fresh medium. After the addition of fresh medium, WST-1 solution was added at $10 \mu \mathrm{L} /$ well. The cells were then incubated for $3 \mathrm{hr}$ at $37^{\circ} \mathrm{C}$, and absorbance was measured at $450 \mathrm{~nm}$ using a microplate reader (Tecan, Männedorf, Switzerland). The cell viability was expressed as a relative percentage of the control viability, after subtraction of the background value.

Detection of intracellular lipid accumulation by oil red $O$ staining and measurement of TG content by subsequent isopropanol extraction. Lipid droplet formation in hepatocytes was monitored by oil red $\mathrm{O}$ staining. AML-12 cells were grown on a 6-well plate and treated with various concentrations of ethanol $(0 \sim 500 \mathrm{mM})$ or ISL $(0 \sim 200 \mu \mathrm{M})$ in fresh medium. The cells were then fixed in 10\% formalin in PBS, rinsed with $60 \%$ isopropanol, and stained with oil red $\mathrm{O}$ solution at room temperature $\left(25^{\circ} \mathrm{C}\right)$ for $30 \mathrm{~min}$. The TG content was quantified by extracting the samples with isopropanol at room temperature for $5 \mathrm{~min}$ and measuring the absorbance at $510 \mathrm{~nm}$. The intracellular TG content was thus measured in AML12 cells.

Immunoblot assay. Samples were prepared from AML-12 cells, which were treated as described above, and subjected to immunoblotting, as described previously (23). The proteins were resolved on a $10 \%$ gel and transferred to polyvinylidine difluoride membrane (PVDF) (Millipore, Bedford, MA, USA). Then membrane was incubated with primary antibodies of SIRT1 (Cell Signaling Technology, 
Canvers, MA, USA), AMPK (Cell Signaling Technology), phospho-AMPK (pAMPK) (Cell Signaling Technology), sterol regulatory element-binding protein-1c (SREBP1c, Santa Cruz Biotechnology, Dallas, TX, USA), peroxisome proliferator-activated receptor alpha (PPAR $\alpha$, Abcam, Cambridge, UK) and $\beta$-actin (Cell Signaling Technology) at $4^{\circ} \mathrm{C}$ for overnight, and secondary antibodies of Mouse IgG (Cell signaling Technology) and Rabbit IgG (Cell signaling Technology) were incubated at room temperature for $1 \mathrm{hr}$. The bands detected using an enhanced chemiluminescence (ECL) system (Amersham, Buckinghamshire, UK).

Data analysis. The data are expressed as the mean \pm standard deviation (SD) and analyzed using SPSS 16.0 software (SPSS, Chicago, IL, USA). The differences among multiple groups were analyzed by one-way analy- sis of variance followed by the Bonferroni post-hoc test. $*, p$-value less than 0.05 was considered statistically significant.

\section{RESULTS}

Treatment with ethanol and ISL influences cell viability in a dose-dependent manner. In order to elucidate the biological pathway affected by ISL in AML-12 cells treated with ethanol, the WST-1 assay was first used to evaluate the effect of ethanol and ISL on cell viability. The viability of AML-12 cells treated with various doses of ethanol $(0 \sim 500 \mathrm{mM})$ and ISL $(0 \sim 200 \mu \mathrm{M})$ was thus determined (Fig. 2). While treatment with $5 \sim 50 \mu \mathrm{M}$ of ISL increased cell proliferation, strong cytotoxicity was observed over $100 \mu \mathrm{M}$ concentrations of ISL, after a treatment period of $24 \mathrm{hr}$ (Fig. 2A). Similarly, the hepatotoxicity of
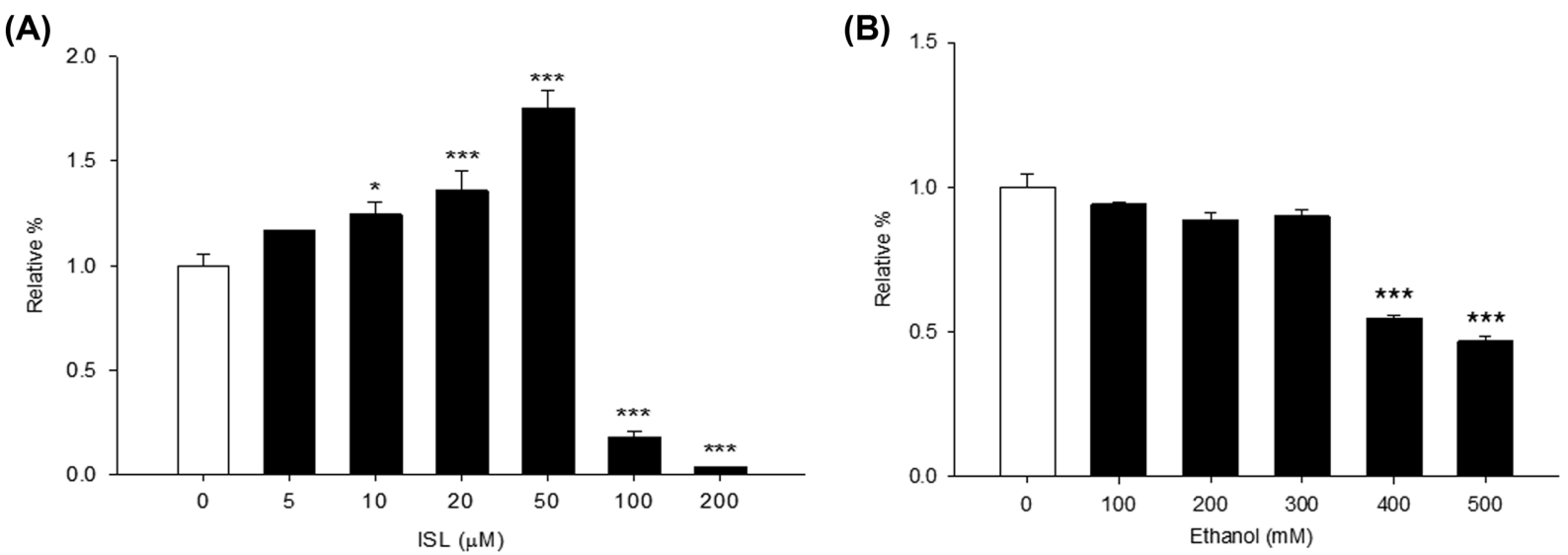

Fig. 2. Dose-dependent cytotoxicity of isoliquiritigenin (ISL) and ethanol. (A) Cytotoxicity after $24 \mathrm{hr}$ ISL treatment. (B) Cell viability after $72 \mathrm{hr}$ ethanol treatment. $p<0.05 ; * 0.001$.

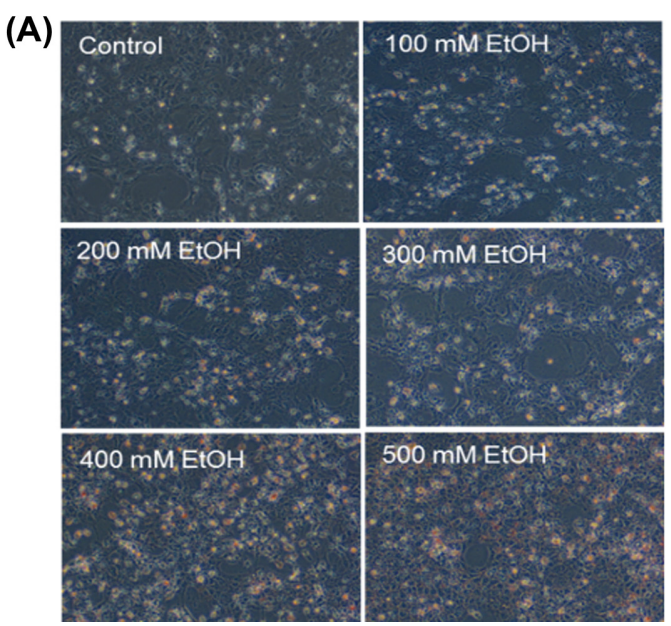

(B)

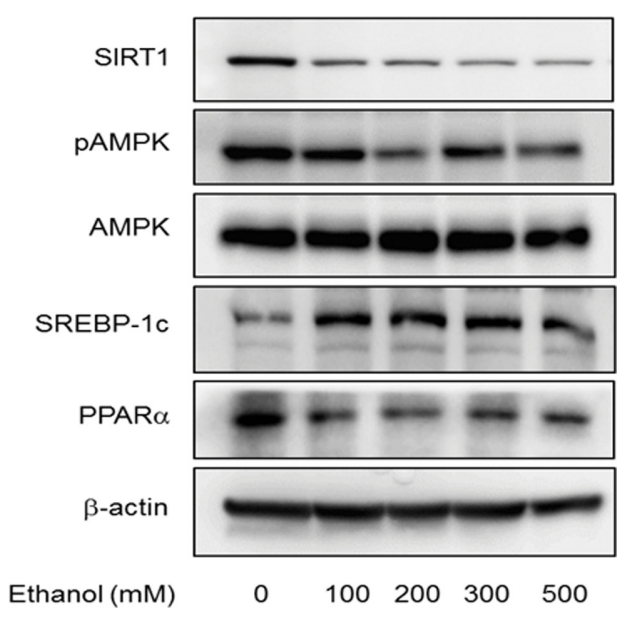

Fig. 3. Dose-dependent effect of $72 \mathrm{hr}$ ethanol treatment on lipid accumulation and expression of downstream proteins in the AMPK-SIRT1 pathway in AML-12 cells. (A) Representative images of oil red O staining ( $\times 200)$. (B) Representative images of western blots showing the effect of $72 \mathrm{hr}$ treatment with $200 \mathrm{mM}$ ethanol on AMPK-SIRT1 signaling. 
ethanol was evaluated by exposing AML-12 cells to various doses of ethanol $(0 \sim 500 \mathrm{mM})$ for $72 \mathrm{hr}$ (Fig. 2B). Compared to untreated cells, the cells treated over 400 $\mathrm{mM}$ concentrations of ethanol showed decreased viability. Therefore, we confirmed that the cytotoxic effect of ISL and ethanol increased in a dose-dependent manner.

Ethanol accelerates lipid accumulation and downregulates SIRT1-AMPK signaling. We exposed AML12 cells to various concentrations of ethanol $(0 \sim 500 \mathrm{mM})$ for $72 \mathrm{hr}$ to induce alcoholic steatosis in vitro and observed the morphological changes between control and ethanoltreated cells (Fig. 3). Ethanol-induced fat accumulation in AML-12 cells was indicated by the oil red $\mathrm{O}$ staining results (Fig. 3A). Hepatic steatosis was observed in all ethanol-treated cells. A greater number of lipid droplets were formed when cells were exposed to over $400 \mathrm{mM}$ of ethanol for $72 \mathrm{hr}$. In order to establish whether ethanol aggravates hepatocyte steatosis via the SIRT1-AMPK pathway, we also measured the expression of downstream proteins in the pathway including SREBP-1c and PPAR $\alpha$ via immunoblotting (Fig. 3B). AMPK phosphorylation and SIRT1 expressions were downregulated and SREBP1c expression was upregulated in AML-12 cells treated with high levels of ethanol, compared to that observed in untreated cells (Fig. 3B). The expression of PPAR $\alpha$, a ligand-activated transcription factor involved in the regulation of hepatic fatty acid oxidation, also dose-dependently decreased in AML-12 cells with ethanol-induced hepatic steatosis. Thus, our data showed that fat accumulation was accelerated and protein expression in the SIRT1-AMPK pathway was downregulated by ethanol exposure.

\section{Elucidation of the mechanism underlying the protec-} tive effects of ISL against ethanol-induced steatosis. To further elucidate the mechanisms underlying the antisteatotic effects of ISL, AML-12 cells were treated with different concentration of ISL $(5,10,20$, and $50 \mathrm{mM})$ and $200 \mathrm{mM}$ ethanol for $72 \mathrm{hr}$. In this study, $200 \mathrm{mM}$ ethanol was selected as the optimal concentration, as only mild
(A)
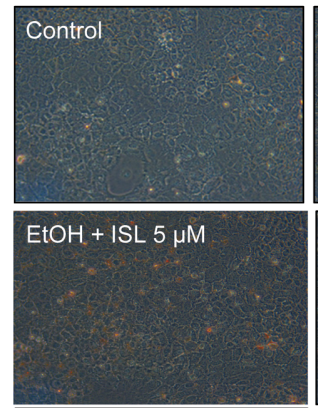

$\mathrm{EtOH}+\mathrm{ISL} 20 \mu \mathrm{M}$

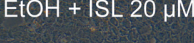

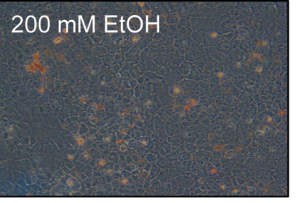

$\mathrm{EtOH}+\mathrm{ISL} 10 \mu \mathrm{M}$

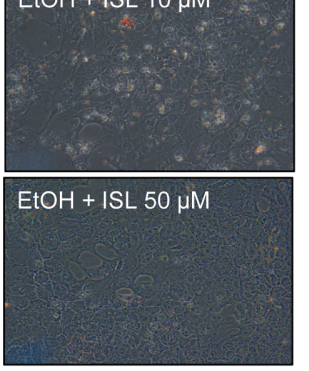

(B)

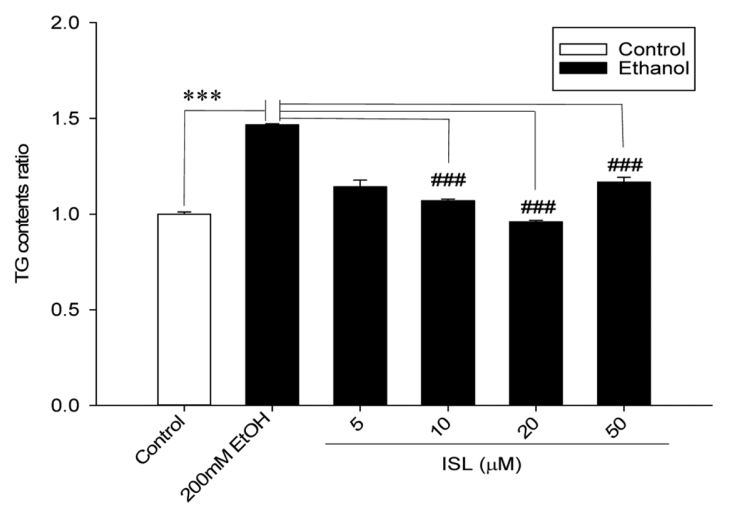

(C)

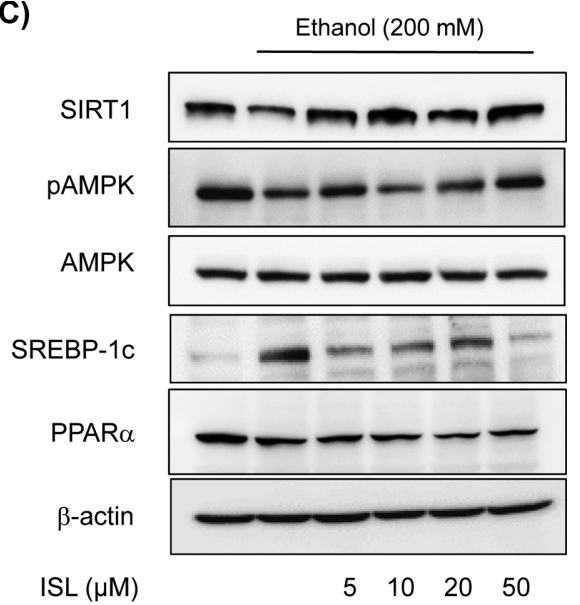

Fig. 4. Effect of isoliquiritigenin (ISL) on AMPK-SIRT1 pathway in AML-12 cells. (A) Representative images of oil red $O$ staining ( $\times 200)$. (B) Bar graph showing the triglyceride content in AML-12 cells after 3-day treatment with a combination of $200 \mathrm{mM}$ ethanol and $0,5,10,20$, or $50 \mu \mathrm{M}$ ISL. (C) Representative images of western blots showing the effect of 3 days treatment with a combination of $200 \mathrm{mM}$ ethanol and $0,5,10,20$, or $50 \mu \mathrm{M}$ ISL on SIRT1-AMPK signaling. ${ }^{* * * *} p<0.001 ;{ }^{\# \# \#} p<0.001$. 


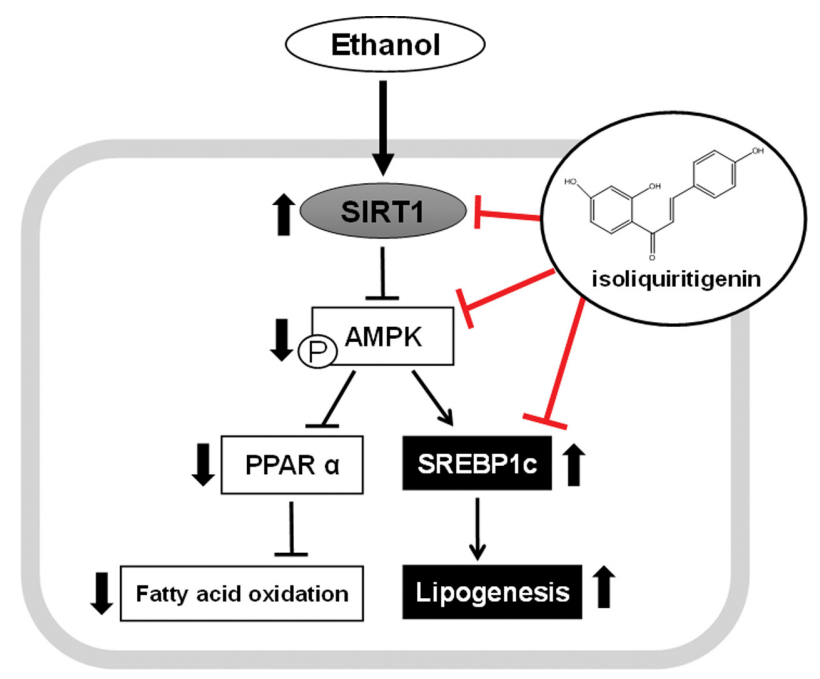

Fig. 5. Proposed signaling pathway. Isoliquiritigenin (ISL) regulated hepatic lipid metabolism under ethanol exposure condition by up-regulating SIRT1-AMPK pathway, which led to dysregulation of the expression of lipogenesis.

hepatotoxicity and no significant decrease in cell viability were observed at that concentration. A change in cell morphology was observed in ethanol and/or ISL-treated cells (Fig. 4A). Although a significant increase in the number of lipid droplets was observed in AML-12 cells treated with $200 \mathrm{mM}$ ethanol alone for $72 \mathrm{hr}$, the number decreased in cells treated with a combination of ethanol and ISL. TG content was also measured after the extraction of oil red $\mathrm{O}$ stain using isopropanol (Fig. 4B). We observed that ethanol exposure caused a 1.5 -fold increase in TG levels, whereas ISL treatment suppressed ethanol-induced lipid accumulation in AML-12 cells.

To further confirm the effects of ISL on the SIRT1AMPK pathway, we examined the expression of SIRT1, AMPK, pAMPK, SREBP-1c, and PPAR $\alpha$ (Fig. 4C). ISL significantly increased the expression of SIRT1 and pAMPK, and decreased SREBP-1c expression in AML-12 cells. However, we could not find significantly recovery effect at the expression of PPAR $\alpha$ with ISL treatment in AML-12 cells. Further, ethanol-induced hepatic steatosis was markedly and dose-dependently inhibited by treatment with ISL.

\section{DISCUSSION}

In summary, ISL treatment effectively ameliorated chronic ethanol consumption-induced fat accumulation and dysregulation of hepatic lipid metabolism by regulating the expression of pAMPK, SIRT1 and SREBP-1c (Fig. 5).

The present study was aimed to investigate the possibility of using ISL for the treatment of liver diseases by evaluating its anti-steatotic effects. Our results showed that
ISL ameliorated ethanol exposure-induced liver lipid deposition by decreasing the TG level and regulating the expression of SREBP-1c in hepatocytes. Moreover, the inhibitory effect of ISL on fat accumulation in AFLD was mediated by the SIRT1-AMPK pathway. Thus, our study suggests that ISL might be a promising therapeutic agent for improving AFLD-induced hepatic damage.

We investigated the dose-dependent viability of AML12 cells after $72 \mathrm{hr}$ ethanol treatment or $24 \mathrm{hr}$ ISL treatment (Fig. 2). There was a significant decrease in cell viability at $400 \mathrm{mM}$ and higher concentrations of ethanol, which is consistent with the MTT assay results reported by Luo et al. (24) in alcohol-damaged hepatocytes. Although ISL could significantly increase the survival rate of hepatocytes in a dose-dependent manner, hepatotoxicity was observed at $100 \mu \mathrm{M}$ and higher concentrations of ISL. In a previous study, $48 \mathrm{hr}$ treatment with $50 \mu \mathrm{M}$ ISL was reported to inhibit the proliferation of a human endometrial cancer cell line (Hec 1A) (25).

The regulation of hepatic signaling plays an important role in controlling hepatic lipid and energy metabolism in response to nutrient availability (2). Dysfunction of these pathways is commonly linked to several ethanol-induced liver diseases. There is increasing evidence that SIRT1 systemically regulates lipid and energy homeostasis in many metabolic tissues (17). Similarly, several functional studies have shown that AMPK is a central regulator of lipid metabolism and glucose homeostasis $(1,10)$. Activated AMPK inhibits fatty acid synthesis and stimulates energy-producing pathways (26). Synergistic activation of AMPK and SIRT1 plays a key role in insulin sensitivity and glycemic control (27). It is also suggested that SIRT1 and AMPK regulate each other, share similar signaling pathways, and regulate many common targets (28). Studies in cultured hepatic cells have provided evidence that SIRT1 is able to stimulate AMPK activity via modulation of liver kinase B1, an upstream AMPK kinase $(12,29)$. Thus, this unique SIRT1-AMPK signaling participates in regulating various lipid metabolism and inflammation pathways in alcoholic liver disease $(12,28,29)$. As an energy source, ethanol induces lipid accumulation and stimulates free fatty acid (FFA) synthesis through activation (30) and/or expression (31) of SREBP-1c, a transcription factor for lipogenic enzymes such as fatty acid synthase.

Our previous studies have adequately established that ethanol dysregulates lipid metabolism and causes hepatocyte injury and hepatic steatosis via the SIRT1-AMPK pathway $(23,32)$. The activation of AMPK/SIRT1 signaling in the liver has been found to increase fatty acid oxidation and repress lipogenesis primarily by modulating the activity of SREBP-1c or PPAR $\gamma$ coactivator- $\alpha$ (PGC-1 $\alpha) /$ PPAR $\alpha(33,34)$. In the current study, we confirmed that AMPK phosphorylation, which significantly decreased upon treatment with ethanol alone (Fig. 3B), was restored upon 
treatment with both ethanol and ISL (Fig. 4C). Moreover, treatment of AML-12 cells with ISL resulted in a complete recovery of the ethanol-induced suppression of SIRT1 and, as evidenced by oil red $\mathrm{O}$ staining, inhibition of ethanol-induced SREBP-1c expression and fat accumulation.

Several studies have demonstrated that acute or chronic ethanol exposure increases SREBP-1c expression and TG content and reduces fatty acid oxidation. SREBP-1c overexpressing mice have been reported to show massive fatty livers, which supports the central role of SREBP-1c in fatty liver development (35). Ethanol also influences hepatic FFA oxidation by regulating PPARs, a family of transcription factors involved in lipid metabolism (36). Although ISL significantly decreased of SREBP-1c expression, increased level by ethanol treatment, ISL didn't show recovery effect of PPAR $\alpha$ expression. The present study strongly implicated ISL as a potent agonist of SIRT1-AMPK signaling in ethanol exposure-induced steatosis following prevent ethanol-induced lipid accumulation through not increasing fatty acid oxidation but blocking lipid synthesis.

So far, no study has reported the inhibitory effect of ISL on fat accumulation in ethanol-induced hepatosteatosis. Our results indicate that the beneficial effect of ISL on alcoholic fatty liver and liver injury may be owing to improvement of homeostatic lipid metabolism in the liver. Therefore, a major finding of the present study is that the protective effect of ISL against hepatic steatosis is mediated by AMPK-SIRT1 signaling and involves suppression of the upregulation of lipid metabolism by lipogenic genes in hepatocytes. In conclusion, our study provides information on the pharmacological effect of ISL on lipid accumulation by utilizing an in vitro model of early alcoholic steatosis. Further studies are required to elucidate the long-term effects of ISL on the more advanced hepatic changes associated with AFLD or nonalcoholic fatty liver disease, such as fibrosis, cirrhosis, and hepatocellular carcinoma development.

\section{ACKNOWLEDGMENTS}

This work was supported by the National Research Foundation of Korea (NRF) grant funded by the Korea government (MSIP) (No. 2015R1A2A2A01004286).

\section{CONFLICT OF INTEREST}

The authors have no conflict of interest to disclose.

Received September 6, 2017; Revised November 11, 2017; Accepted November 27, 2017

\section{REFERENCES}

1. O'Shea, R.S., Dasarathy, S. and McCullough, A.J. (2010)
Alcoholic liver disease. Am. J. Gastroenterol., 105, 14-32.

2. Watanabe, Y., Nagai, Y., Honda, H., Okamoto, N., Yamamoto, S., Hamashima, T., Ishii, Y., Tanaka, M., Suganami, T., Sasahara, M., Miyake, K. and Takatsu, K. (2016) Isoliquiritigenin attenuates adipose tissue inflammation in vitro and adipose tissue fibrosis through inhibition of innate immune responses in mice. Sci. Rep., 6, 23097.

3. Gao, B. and Bataller, R. (2011) Alcoholic liver disease: pathogenesis and new therapeutic targets. Gastroenterology, 141, 1572-1585.

4. Nagy, L.E., Ding, W.X., Cresci, G., Saikia, P. and Shah, V.H. (2016) Linking pathogenic mechanisms of alcoholic liver disease with clinical phenotypes. Gastroenterology, 150, 1756-1768.

5. Lieber, C.S., Leo, M.A., Wang, X. and Decarli, L.M. (2008) Effect of chronic alcohol consumption on Hepatic SIRT1 and PGC-1alpha in rats. Biochem. Biophys. Res. Commun., 370, 44-48.

6. Lu, Y. and Cederbaum, A.I. (2008) CYP2E1 and oxidative liver injury by alcohol. Free. Radic. Biol. Med., 44, 723-738.

7. Purushotham, A., Xu, Q. and Li, X. (2012) Systemic SIRT1 insufficiency results in disruption of energy homeostasis and steroid hormone metabolism upon high-fat-diet feeding. FASEB J., 26, 656-667.

8. Shen, Z., Ajmo, J.M., Rogers, C.Q., Liang, X., Le, L., Murr, M.M., Peng, Y. and You, M. (2009) Role of SIRT1 in regulation of LPS- or two ethanol metabolites-induced TNFalpha production in cultured macrophage cell lines. Am. J. Physiol. Gastrointest. Liver Physiol., 296, G1047-G1053.

9. Ajmo, J.M., Liang, X., Rogers, C.Q., Pennock, B. and You, M. (2008) Resveratrol alleviates alcoholic fatty liver in mice. Am. J. Physiol. Gastrointest. Liver Physiol., 295, G833-G842.

10. Shen, Z., Liang, X., Rogers, C.Q., Rideout, D. and You, M. (2010) Involvement of adiponectin-SIRT1-AMPK signaling in the protective action of rosiglitazone against alcoholic fatty liver in mice. Am. J. Physiol. Gastrointest. Liver Physiol., 298, G364-G374.

11. Everitt, H., Hu, M., Ajmo, J.M., Rogers, C.Q., Liang, X., Zhang, R., Yin, H., Choi, A., Bennett, E.S. and You, M. (2013) Ethanol administration exacerbates the abnormalities in hepatic lipid oxidation in genetically obese mice. Am. J. Physiol. Gastrointest. Liver Physiol., 304, G38-G47.

12. Lan, F., Cacicedo, J.M., Ruderman, N. and Ido, Y. (2008) SIRT1 modulation of the acetylation status, cytosolic localization, and activity of LKB1. Possible role in AMP-activated protein kinase activation. J. Biol. Chem., 283, 2762827635.

13. Lin, H., Liu, J. and Zhang, Y. (2011) Developments in cancer prevention and treatment using traditional Chinese medicine. Front. Med., 5, 127-133.

14. Cao, Y., Wang, Y., Ji, C. and Ye, J. (2004) Determination of liquiritigenin and isoliquiritigenin in Glycyrrhiza uralensis and its medicinal preparations by capillary electrophoresis with electrochemical detection. J. Chromatogr. A, 9, 1-2.

15. Haraguchi, H., Ishikawa, H., Mizutani, K., Tamura, Y. and Kinoshita, T. (1998) Antioxidative and superoxide scavenging activities of retrochalcones in Glycyrrhiza inflata. Bioorg. Med. Chem., 6, 339-347. 
16. Tawata, M., Aida, K., Noguchi, T., Ozaki, Y., Kume, S., Sasaki, H., Chin, M. and Onaya, T. (1992) Anti-platelet action of isoliquiritigenin, an aldose reductase inhibitor in licorice. Eur. J. Pharmacol., 212, 87-92.

17. Yu, S.M. and Kuo, S.C. (1995) Vasorelaxant effect of isoliquiritigenin, a novel soluble guanylate cyclase activator, in rat aorta. Br. J. Pharmacol., 114, 1587-1594.

18. Tamir, S., Eizenberg, M., Somjen, D., Izrael, S. and Vaya, J. (2001) Estrogen-like activity of glabrene and other constituents isolated from licorice root. J. Steroid. Biochem. Mol. Biol., 78, 291-298.

19. Lee, Y.K., Chin, Y.W., Bae, J.K., Seo, J.S. and Choi, Y.H. (2013) Pharmacokinetics of isoliquiritigenin and its metabolites in rats: low bioavailability is primarily due to the hepatic and intestinal metabolism. Planta. Med., 79, 16561665.

20. Ii, T., Satomi, Y., Katoh, D., Shimada, J., Baba, M., Okuyama, T., Nishino, H. and Kitamura, N. (2004) Induction of cell cycle arrest and p21(CIP1/WAF1) expression in human lung cancer cells by isoliquiritigenin. Cancer Lett., 207, 27-35.

21. Wang, Z., Wang, N., Liu, P., Chen, Q., Situ, H., Xie, T., Zhang, J., Peng, C., Lin, Y. and Chen, J. (2014) MicroRNA25 regulates chemoresistance-associated autophagy in breast cancer cells, a process modulated by the natural autophagy inducer isoliquiritigenin. Oncotarget, 5, 7013-7026.

22. Lee, H.K., Yang, E.J., Kim, J.Y., Song, K.S. and Seong, Y.H. (2012) Inhibitory effects of Glycyrrhizae radix and its active component, isoliquiritigenin, on Abeta(25-35)-induced neurotoxicity in cultured rat cortical neurons. Arch. Pharm. Res., 35, 897-904.

23. Kim, S.J., Ki, S.H. and Lee, S. (2015) Protective effects of korean red ginseng against alcohol-induced hepatosteatosis. J. Life Sci., 25, 317-322.

24. Luo, H., Cao, R., Wang, L. and Zhu, L. (2016) Protective effect of Cistanchis A on ethanol-induced damage in primary cultured mouse hepatocytes. Biomed. Pharmacother., 83, 1071-1079.

25. Kim, Y.G., Ramachandran, S., Kim, D.C., Hong, Y.B., Kim, E.H., Kwon, S.H., Shin, S.J., Cha, S.D., Bae, I. and Cho, C.H. (2010) Isoliquiritigenin inhibited cell proliferation and triggered apoptosis in human endometrial cancer cell Line. J. Womens Med., 3, 89-95.

26. Dagon, Y., Mantzoros, C.S. and Kim, Y.B. (2016) AMPK $\leftrightarrow$
Sirt1: from a signaling network to a combination drug. Metabolism, 65, 1692-1694.

27. Banerjee, J., Bruckbauer, A. and Zemel, M.B. (2016) Activation of the AMPK/Sirt1 pathway by a leucine-metformin combination increases insulin sensitivity in skeletal muscle, and stimulates glucose and lipid metabolism and increases life span in Caenorhabditis elegans. Metabolism, 65, 16791691.

28. Ruderman, N.B., Xu, X.J., Nelson, L., Cacicedo, J.M., Saha, A.K., Lan, F. and Ido, Y. (2010) AMPK and SIRT1: a longstanding partnership? Am. J. Physiol. Endocrinol. Metab., 298, E751-E760

29. Hou, X., Xu, S., Maitland-Toolan, K.A., Sato, K., Jiang, B., Ido, Y., Lan, F., Walsh, K., Wierzbicki, M., Verbeuren, T.J., Cohen, R.A. and Zang, M. (2008) SIRT1 regulates hepatocyte lipid metabolism through activating AMP-activated protein kinase. J. Biol. Chem., 283, 20015-20026.

30. Kalaany, N.Y. and Mangelsdorf, D.J. (2006) LXRS and FXR: the yin and yang of cholesterol and fat metabolism. Annu. Rev. Physiol., 68, 159-191.

31. Yin, H.Q., Kim, M., Kim, J.H., Kong, G., Kang, K.S., Kim, H.L., Yoon, B.I., Lee, M.O. and Lee, B.H. (2007) Differential gene expression and lipid metabolism in fatty liver induced by acute ethanol treatment in mice. Toxicol. Appl. Pharmacol., 223, 225-233.

32. Han, J.Y., Lee, S., Yang, J.H., Kim, S., Sim, J., Kim, M.G., Jeong, T.C., Ku, S.K., Cho, I.J. and Ki, S.H. (2015) Korean Red Ginseng attenuates ethanol-induced steatosis and oxidative stress via AMPK/Sirt1 activation. J. Ginseng Res., 39, 105-115.

33. Canto, C. and Auwerx, J. (2009) PGC-1alpha, SIRT1 and AMPK, an energy sensing network that controls energy expenditure. Curr. Opin. Lipidol., 20, 98-105.

34. Shen, Z., Liang, X., Rogers, C.Q., Rideout, D. and You, M. (2010) Involvement of adiponectin-SIRT1-AMPK signaling in the protective action of rosiglitazone against alcoholic fatty liver in mice. Am. J. Physiol. Gastrointest. Liver Physiol., 298, G364-G374.

35. Liu, J. (2014) Ethanol and liver: recent insights into the mechanisms of ethanol-induced fatty liver. World J. Gastroenterol., 20, 14672-14685.

36. Donohue, T.M., Jr. (2007) Alcohol-induced steatosis in liver cells. World J. Gastroenterol., 13, 4974-4978. 